\title{
Multidimensional upwind schemes based on fluctuation-splitting for systems of conservation laws
}

\author{
H. Deconinck ${ }^{1}$, H. Paillère ${ }^{1}$, R. Struijs ${ }^{1}$, and P. L. Roe ${ }^{2}$ \\ 1 CFD-Group, von Karman Institute for Fluid Dynamics \\ 2 Aerospace Department, The University of Michigan
}

\begin{abstract}
A class of truly multidimensional upwind schemes for the computation of inviscid compressible flows is presented here, applicable to unstructured cell-vertex grids. These methods use very compact stencils and produce sharp resolution of discontinuities with no overshoots.
\end{abstract}

\section{Introduction}

In this contribution we survey a class of upwind schemes for hyperbolic conservation laws on unstructured grids consisting of triangles or tetrahedra, and based on the concept of fluctuation or residual splitting. Unlike standard upwind schemes which are based on a one-dimensional physical model (the Riemann problem), the methods discussed here use a multidimensional physical model for the upwinding. They do not fit in a standard Finite Volume approach with piecewise continuous representation of the unknowns. In this respect they are much closer to Finite Element methods based on linear elements, with which they share a continuous piecewise linear representation over the triangles. On the other hand, they share with standard upwind methods the properties of non-symmetric stencils and control of monotonicity over discontinuities, and they can be considered as truly multidimensional generalizations of the successful TVD upwind methods developed during the eighties.

\section{Concept of fluctuation-splitting}

In this section, we recall the fluctuation-splitting formulation as it was initially proposed in 1981 , (Roe 1981, 1982). Contrary to what has been done in later interpretations, this formulation does not necessarily appeal to Finite Volumes nor to the Riemann problem. Given the solution at a certain time level $n$ as a discrete mesh function $\left(\mathbf{u}_{i}, i=1, \ldots, N\right\}$ on an irregular grid with meshpoints $\left\{x_{i}\right\}$, one assumes a continuous piecewise linear representation in between the meshpoints, precisely as when using linear Finite Elements in space. Once this view-point has been taken there is no room left for Riemann problems, since the initial data are continuous. An upwind space discretization is obtained as follows. Consider first a nonlinear scalar conservation equation

$u_{t}+F_{x}=0$,

with advection speed $\lambda=F_{u}$. Assuming piecewise linear initial data at time level $n$ allows to define linear wave solutions for each cell $\left[x_{i}, x_{i+1}\right]$ with length $\Delta x_{i+1 / 2}=x_{i+1}-x_{i}$. Such wave solutions are of the form

$u(x, t)=u_{i}^{n}+\frac{u_{i+1}^{n}-u_{i}^{n}}{x_{i+1}-x_{i}}\left(x-x_{i}-\bar{\lambda}\left(t-t^{n}\right)\right)$,

where $\bar{\lambda}$ is a suitable averaged speed over the interval. If no information is used from neighbouring 


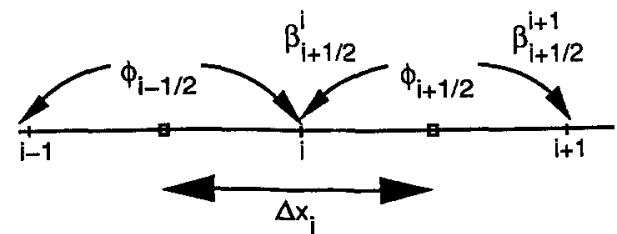

Fig. 1. Fluctuation-splitting scheme in 1D

cells, solution (2) contains the maximum of information about the time evolution for this cell. The following strategy for updating the vertices of the cell is then equivalent to first order upwinding and Euler explicit timestepping. Suppose that the averaged speed is positive. Then at $t^{n+1}=t^{n}+\Delta t$, the linear initial value distribution has shifted to the right over a distance $\bar{\lambda} \Delta t$. Hence, the solution $u_{i}$ at the left is not affected, while the solution $u_{i+1}$ at the right is changed according to (2). Conversely, for an averaged speed which is negative, the linear initial value distribution has shifted to the left and the solution $u_{i+1}$ at the right is not affected. Taking into account the non-uniformity of the mesh, this leads to the following updating scheme for meshpoint $i$, by adding the contributions from the two neighbouring cells, denoted by the subscripts $i+\frac{1}{2}$ and $i-\frac{1}{2}$, Fig. 1,

$u_{i}^{n+1}=u_{i}^{n}+\frac{\Delta t}{\Delta x_{i}}\left(\beta_{i+1 / 2}^{i} \Phi_{i+1 / 2}+\beta_{i-1 / 2}^{i} \Phi_{i-1 / 2}\right)$

where $\Delta x_{i}=\frac{1}{2}\left(\Delta x_{i+1 / 2}+\Delta x_{i-1 / 2}\right)$ is the median dual cell around $x_{i}$ and $\Phi_{i+1 / 2}$ is the cell residual or cell-fluctuation for a cell, given by

$\Phi_{i+1 / 2}=-\bar{\lambda}\left(u_{i+1}^{n}-u_{i}^{n}\right)$.

The downstream distribution coefficients $\beta$ summing up to one for a given cell, are defined as follows:

$\beta_{i+1 / 2}^{i}=0, \quad \beta_{i+1 / 2}^{i+1}=1 \quad$ for $\bar{\lambda} \geqq 0$

$\beta_{i+1 / 2}^{i}=1, \quad \beta_{i+1 / 2}^{i+1}=0$ for $\bar{\lambda}<0$.

Here, the superscripts $i$ and $i+1$ refer to the nodes of element $i+\frac{1}{2}$. Substituting in Eq. (3) one obtains standard first order upwinding. We will call the scheme positive if the coefficients of all unknowns in the right-hand side of (3) are positive. Since these coefficients sum up to one, such a scheme leads to an update which is bounded by the minimum and maximum of the stencil at $t=t^{n}$, which is a sufficient condition for stability and monotonic discontinuity capturing. For scheme (5), this leads to the well-known CFL condition:

$\Delta t_{i} \leqq \frac{\Delta x_{i}}{\max \left(0, \bar{\lambda}_{i-1 / 2}\right)+\max \left(0,-\bar{\lambda}_{i+1 / 2}\right)}$.

For arbitrary definition of the linearized advection speed $\bar{\lambda}$ the above schemes will not satisfy discrete conservation. However, discrete conservation is easely ensured by imposing a constraint on the linearization. Indeed, summing up Eq. (3) for all meshpoint one obtains cancelling of all fluxes at the interior cell boundaries provided that

$F_{i+1}-F_{i}=\bar{\lambda}\left(u_{i+1}-u_{i}\right)$,

which is of course the standard Roe-linearization. A procedure suitable for generalization in two and three space dimensions is the following: make use of the assumption that $u$ has linear variation in space and redefine the fluctuation as the contour integral of the fluxes over the cell,

$\Phi_{i+1 / 2}=-\int_{x_{i}}^{x_{2}+1} F_{x}(u(x)) d x=-[F]_{i}^{i+1}$.

Using the chain rule and taking $u_{x}$ out of the integration leads to the generalization

$\bar{\lambda}=\frac{1}{\Delta x_{i+1 / 2}} \int_{x_{i}}^{x_{i}+1} F_{u}(u(x)) d x$. 
The fluctuation splitting concept allows an easy way to analyse the shock capturing properties of the scheme (Roe 1982): For example, consider a steady shock captured over the mesh, such that $F\left(u_{0}\right)=F\left(u_{N}\right)$ but $u_{0} \neq u_{N}$. The $\bar{\lambda}$ have opposite signs on both sides and will send the information towards the middle of the domain. Capturing of the shock will occur in a layer of one or two cells: The case of one cell occurs if $\bar{\lambda}=0$ for the cell separating the left and right states, such that at steady state all cells are in equilibrium individually. To show the existence of capturing with one intermediate point, suppose an intermediate state $u_{m}$, and assume speeds on both sides that are opposite and non zero, pointing towards $x_{m}$. Equilibrium at $x_{m}$ is then obtained for $u_{m}$ solution of

$\Phi_{m+1 / 2}+\Phi_{m-1 / 2}=-\bar{\lambda}_{m+1 / 2}\left(u_{N}-u_{m}\right)-\bar{\lambda}_{m-1 / 2}\left(u_{m}-u_{0}\right)=0$.

Because of the existence of shocks captured over one cell, the scheme is not entropy-satisfying: The scheme accepts as a steady state also the situation where the two speeds point away from the shock cell which itself is in equilibrium. As discussed in Roe (1982) a two-cell capturing as with Oshers Riemann solver is always entropy satisfying. This will prove an important consideration in the multidimensional generalization.

\section{Scalar conservation laws in $2 D$}

The generalization of the concepts introduced before to two space dimensions is straight forward. Consider the scalar conservation equation

$u_{t}+\nabla \cdot \boldsymbol{F}(u)=0, \quad \boldsymbol{F}=F \mathbf{1}_{x}+G \mathbf{1}_{y}$,

with advection speed vector

$\lambda=F_{u} \mathbf{1}_{x}+G_{u} \mathbf{1}_{y}$.

The assumption of continuous, piecewise linear space variation requires triangular cells in $2 \mathrm{D}$, with the solution stored at the vertices. Considering a triangle $T$, the initial value problem is defined by the constant gradient $\nabla u^{n}$ at time $t^{n}$, and the solution evolves in time according to

$u(x, t)=u_{0}+\nabla u^{n} \cdot(x-\bar{\lambda} t)$,

where $\bar{\lambda}$ is a suitable averaged advection speed vector over the cell, determined such that discrete conservation is ensured. As before, we will use the maximum of information contained in (13) to update the nodes of the cell. This is achieved by splitting the cell residual in parts which will be distributed towards the nodes of that particular cell. Hence, the following scheme is the natural generalization of (3) towards two space dimensions:

$u_{i}^{n+1}=u_{i}^{n}+\frac{\Delta t}{S_{i}} \sum_{T} \beta_{T}^{i} \Phi_{T}$

where the summation is carried out over all the cells having $i$ as a common vertex. The surface $S_{i}$ generalizes the median dual edge $\Delta x_{i}=\frac{1}{2}\left(\Delta x_{i+1 / 2}+\Delta x_{i-1 / 2}\right)$ to one third of the area of the surrounding triangles. The coefficients $\beta$ for a given cell $T$ satisfy:

$\sum_{k=1}^{3} \beta_{T}^{i(k)}=1$

where $\{i(k), k=1, \ldots, 3\}$ are the nodes of element $T$. The fluctuation in conservative form $\Phi_{T}$ is a straight forward generalization of (8), obtained by computing the flux balance over a cell, supposing linear variation of $u$ :

$\Phi_{T}=-\oint_{\partial T} \boldsymbol{F} \cdot \boldsymbol{n} d l=-\int_{S_{T}} \nabla \cdot \boldsymbol{F}(u(\boldsymbol{x})) d S$.

Applying the chain rule $F_{x}=F_{u} u_{x}, G_{y}=G_{u} u_{y}$, one obtains the definition of the averaged advection 
speed which assures discrete conservation:

$\bar{\lambda}=\bar{\lambda}_{x} \mathbf{1}_{x}+\bar{\lambda}_{y} \mathbf{1}_{y}$

where as in (9)

$\bar{\lambda}_{x}=\frac{1}{S_{T}} \int_{S_{T}} F_{u}(u(x)) d S \quad \bar{\lambda}_{y}=\frac{1}{S_{T}} \int_{S_{T}} G_{u}(u(x)) d S$.

Using this definition, the conservative fluctuation (16), can be rewritten in the linearized form

$\Phi_{T}=-S_{T} \bar{\lambda} \cdot \nabla u=-\sum_{i=1}^{3} k_{i} u_{i}$

where the summation is over the nodes of the element $T$. The coefficients $k_{i}$ are computed as:

$k_{i}=\frac{1}{2} \bar{\lambda} \cdot n_{i} \quad$ satisfying $\quad \sum_{i=1}^{3} k_{i}=0$,

where $\boldsymbol{n}_{i}$ is the inward scaled normal to the face opposed to vertex $i$. The only remaining ingredient needed to define the fluctuation splitting schemes completely is the precise definition of the coefficients $\beta$ satisfying the constraint (15). Here, we cannot longer rely on any $1 \mathrm{D}$ theory, and a new theory of advection schemes for simplices in 2D has been necessary. This theory is strikingly elegant and is now fairly complete. It is not possible to go in all the details in the context of this paper, and the reader is referred to Roe (1987) and Struijs et al. (1991) for the full details, and to Bourgois et al. (1992) for the generalizations of the schemes to 3D.

First we will define upstream and downstream vertices for a triangle. Consistent with the definition in 1D we define a downstream vertex as a vertex opposite to an inflow face. For such a vertex one always has $k_{i} \geqq 0$. Conversely, an upstream vertex is a vertex for which $k_{i} \leqq 0$. One then observes that there are two different types of triangles: triangles with one or two downstream vertices, see Fig. 2. Having defined upstream and downstream vertices, we can give a consistent definition of an upwind scheme: Recalling (13), we will denote a scheme as an upwind scheme if in accordance with this solution no contribution is sent to the upstream vertices. Hence, we will consider one and two target distributions for triangles. Investigating the possible choices for linear schemes (defined by schemes of the form (14) for which the update is a linear function of the $u_{i}$ if $\bar{\lambda}$ is a constant vector), two different classes appear:

i. A first class in which the coefficients $\beta$ are independent of the $u_{i}$. For this class, a triangle will send no updates to its vertices if it is in equilibrium, corresponding to $k$-exactness for linear polynomial solutions $(k=1)$. On regular grids, this corresponds to a steady state which is second order in space.

ii. A second class in which the coefficients $\beta$ do depend on the unknowns in the following way:

$\beta_{T}^{i}=\frac{\gamma_{T}^{i}}{\Phi_{T}}$ with $\sum_{k=1}^{3} \gamma_{T}^{i(k)}=\Phi_{T}$

where $\gamma_{T}^{i}$ are linear functions of the $u_{j}$.

Substituting in (14), the schemes in this class can be written as:

$u_{i}^{n+1}=u_{i}^{n}+\frac{\Delta t}{S_{i}} \sum_{T} \gamma_{T}^{i}$

The second class allows a cell to be in equilibrium while sending non zero contributions to the downstream vertices whose sum vanishes. Such a scheme will in general only preserve a constant steady state and destroy a linear steady state satisfying $\lambda \cdot \nabla u=0$ for each triangle. These schemes will be called first order in the present context. It turns out that only in this class one can construct linear positive schemes, thus providing a generalized Godunov type theorem in two and three 
space dimensions: positivity and $k$-exactness for linear polynomials are incompatible for linear schemes, Struijs et al. (1991).

Hence, to obtain schemes which are both accurate and positive, it will be necessary to consider nonlinear advection schemes as was done in 1D when TVD properties were introduced. This will be introduced in Sect. 3.2. First, we present in the next section two linear schemes, one for each of the two classes. Other schemes are detailed in Struijs et al. (1991).

\subsection{Linear distribution schemes}

Low Diffusion A scheme (LDA)

A first scheme which is $k$-exact for linear polynomials has been called the "Low Diffusion scheme A" LDA in (Struijs et al. 1991). Here we give a generalized expression of the distribution coefficients, valid both on $2 \mathrm{D}$ triangles $(d=2)$ and 3D tetrahedra $(d=3)$, Bourgois et al. (1992):

$\beta_{T}^{i}=\frac{\max \left(0, k_{i}\right)}{\sum_{j=1}^{d+1} \max \left(0, k_{j}\right)} i=1, \ldots, d+1$.

The scheme will preserve an exact steady state which satisfies $\bar{\lambda} \cdot \nabla u^{n}=0$ because the coefficients are independent of $u_{j}$. If there is only one downstream vertex, the entire residual is sent to that vertex. If there is more than one downstream vertex, there is a nice geometric interpretation: for example in 2D (Fig. 2), the velocity placed at the upstream vertex divides the triangle in two subtriangles. The coefficients $\beta$ for the two downstream vertices are then proportional to the surface of the opposite subtriangle. It is easy to check that scheme (23) is not positive, and that it produces oscillations around discontinuities.

The N-scheme

A second linear scheme belongs to class ii and satisfies (21). It is studied extensively in Roe (1987) and Struijs et al. (1991) for the 2D case. The generalization valid in $2 \mathrm{D}$ and in $3 \mathrm{D}$ is obtained if the coefficient $\gamma$ needed in (21) is defined as follows:

$\gamma_{T}^{i}=\frac{\max \left(0, k_{i}\right)}{\sum_{j=1}^{d+1} \max \left(0, k_{j}\right)} \sum_{j=1}^{d+1}\left[\min \left(0, k_{j}\right)\left(u_{i}^{n}-u_{j}^{n}\right)\right]$.

For a one inflow triangle, the formula is both positive and linearity preserving, since the whole fluctuation is sent to the downstream node. However, for two-inflow triangles, the scheme

$u_{1}^{n+1}:=u_{1}^{n}-\frac{\Delta t}{S_{1}} k_{1}\left(u_{1}^{n}-u_{3}^{n}\right), \quad u_{2}^{n+1}:=u_{2}^{n}-\frac{\Delta t}{S_{2}} k_{2}\left(u_{2}^{n}-u_{3}^{n}\right)$

does not preserve a linear steady state because the differences $\left(u_{i}^{n}-u_{j}^{n}\right)$ can be non zero even when the sum of the $\gamma$ 's vanishes for a triangle in equilibrium. On the other hand, because we

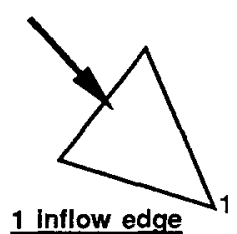

$\beta_{1}=1$

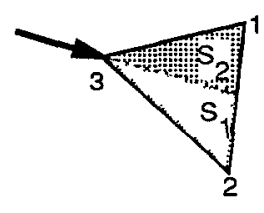

2 inflow edges

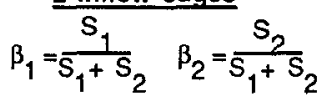

Fig. 2. Graphical interpretation of the LDA scheme in 2D 


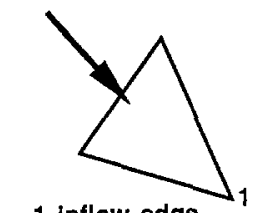

1 inflow edge

$s_{1} u_{1}^{n+1}=s_{1} u_{1}^{n}+\Delta t \phi$

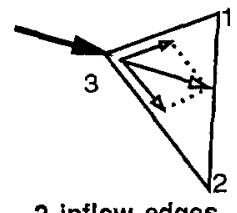

2 inflow edges

$s_{1} u_{1}^{n+1}=s_{1} u_{1}+\Delta t \phi_{31}$

$S_{2} u_{2}^{n+1}=S_{2} u_{2}^{n}+\Delta t \phi_{32} \quad$ Fig. 3. Graphical interpretation of the $N$ scheme in 2D

have complete control over the sign of each coefficient, this scheme is positive for timesteps small enough: The condition can be simplified to a CFL like condition for the local timestep at meshpoint $i$

$\Delta t_{i} \leqq \frac{S_{i}}{\sum_{T} \max \left(0, k_{i}^{T}\right)}$.

As for the previous scheme, the $\mathrm{N}$ scheme has a simple geometric interpretation, see Fig. 3 . This interpretation decomposes the advection speed vector in components parallel to the edges pointing from upstream to downstream vertices. With each component corresponds a one inflow configuration and the resulting fluctuation is sent to the single downstream node.

In Struijs et al. (1991) it is shown that the $\mathrm{N}$-scheme is the optimum linear positive scheme on the compact stencil of simplices meeting at a given vertex, in the sense that among the linear positive schemes of the form (14) it is the one which allows the largest timestep and has the smallest cross diffusion.

\subsection{Nonlinear distribution schemes}

None of the above linear schemes can be positive and $k$-exact at the same time, and as in 1D, nonlinear schemes have to be used for high resolution and non-oscillatory discontinuity capturing. The mechanism by which the nonlinearity is introduced in 1D and in the dimension by dimension extensions relies on nonlinear averaging functions, known as limiters. This usually leads to a widening of the stencil, which is inevitable in 1D. In multidimensional space, one can hope to introduce the nonlinearity while still maintaining a compact stencil. This was first recognized for 2D structured grids by Sidilkover (1990). A remarkable discovery when studying the multidimensional fluctuation splitting schemes was the existence of an alternative for limiting: by looking at the multidimensional scalar advection equation and the wave solution (13), a new mechanism for introducing nonlinearity in the scheme became apparent, which is completely different from the limiting approach and which does not exist in 1D because solution gradients and advection speeds are always in the same direction. Purely at the level of the linear wave solution (13) we can define a "frontal" advection speed vector in the direction of $\nabla u^{n}$, as is well known, Fig. 4. This speed is given by

$\lambda_{m}=\frac{\bar{\lambda} \cdot \nabla u^{n}}{\left|\nabla u^{n}\right|} \boldsymbol{m}=\frac{S_{i} \Phi_{T}}{\left|\nabla u^{n}\right|} \boldsymbol{m}=\lambda_{m} \boldsymbol{m}$,

where

$\boldsymbol{m}=\frac{\nabla u^{n}}{\left|\nabla u^{n}\right|}$ and $\quad \lambda_{m}=\bar{\lambda} \cdot \boldsymbol{m}$.

It is fully legitimate to use the "frontal" speed $\lambda_{m}$ instead of the advection speed $\bar{\lambda}$ in any of the distribution schemes since it does not affect the residual $\left(\bar{\lambda} \cdot \nabla u^{n}=\lambda_{m} \cdot \nabla u^{n}\right)$ and still corresponds to 


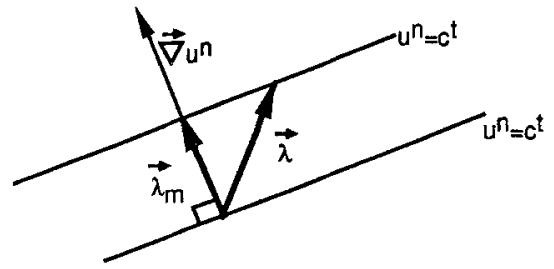

Fig. 4. Frontal and ray speed vectors in $2 \mathrm{D}$
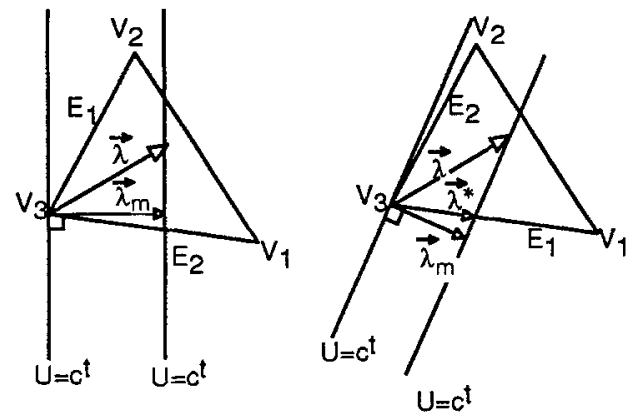

Fig. 5. The NN scheme

the linear wave solution (13) rewritten as

$u(\boldsymbol{x}, t)=u_{0}+\left|\nabla u^{n}\right|\left(\boldsymbol{x} \cdot \boldsymbol{m}-\lambda_{\boldsymbol{m}} t\right)$.

However, using the frontal speed for the definition of the $k_{i}$ makes the scheme nonlinear since these coefficients now depend on $\nabla u^{n}$. Moreover, any of the schemes based on the frontal speed becomes linearity preserving since the frontal speed $\lambda_{m}$ and hence all the $k_{i}$ vanish for a triangle which is in equilibrium. Hence, the two-target formula of the $\mathrm{N}$-scheme (25) discussed in the previous section becomes $k$-exact for linear polynomials when using the frontal speeds.

The resulting scheme is one of a number of nonlinear schemes which have very similar properties. They have been called "NN schemes", standing for Nonlinear N-scheme (Fig. 5). Summarizing, the scheme in 2D is given by the following algorithm:

i. If the triangle has one inflow side according to $\bar{\lambda}$, send the residual to the unique downstream vertex.

ii. If the triangle is two target according to $\bar{\lambda}$, say with nodes 1 and 2 , compute $\lambda_{m}$

1. if both $\lambda_{m} n_{1}>0$ and $\lambda_{m} n_{2}>0$ use the two target formula (25), with $k_{1}^{m}$ and $k_{2}^{m}$ based on the frontal speed.

2. else if $\lambda_{m} n_{1}<0$ and $\lambda_{m} n_{2}>0$ send the residual to node 2 .

3 . else send the residual to node 1 .

It is clear that a steady contact or shear discontinuity aligned with the grid will be preserved over one cell. Indeed, in this case all cells are in equilibrium individually because the gradients are normal to the advection speed vector.

\subsection{A non-linear conservation law}

The shock capturing properties of the NN scheme are studied here, in the case of a 2D non-linear conservation law similar to Burger's equation:

$u_{t}+\lambda \cdot \nabla u=0 \quad \lambda=F_{u}=\left(\begin{array}{c}u \\ 1\end{array}\right) \quad F=\left(\begin{array}{c}u^{2} / 2 \\ u\end{array}\right)$

where $\lambda$ is the advection-speed. Assuming $u$ varies linearly over each cell, $\nabla u$ is a constant, and $\lambda$ being linear in $u$, the fluctuation is given by (19), where the averaged speed is just the arithmetic 
6
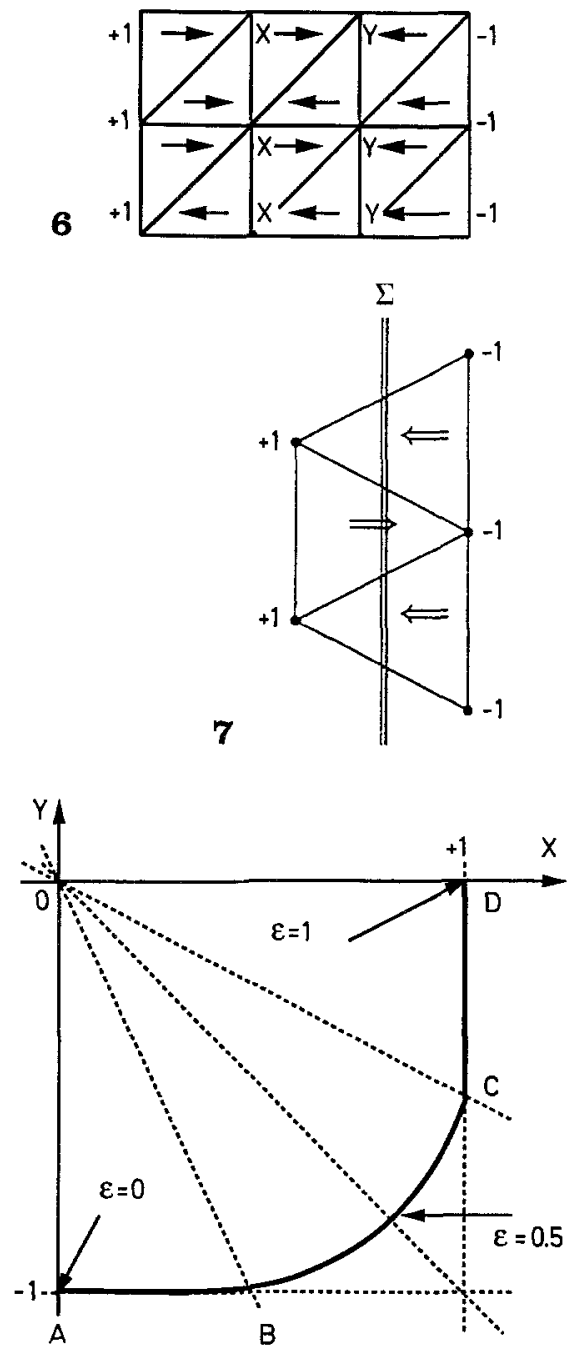

8

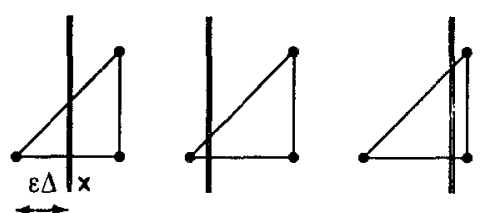

9
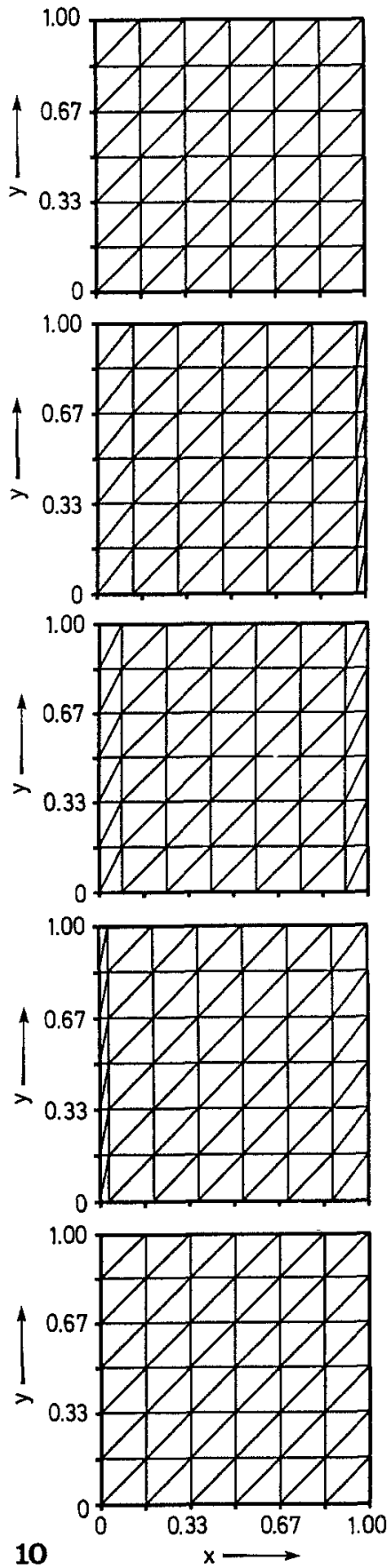
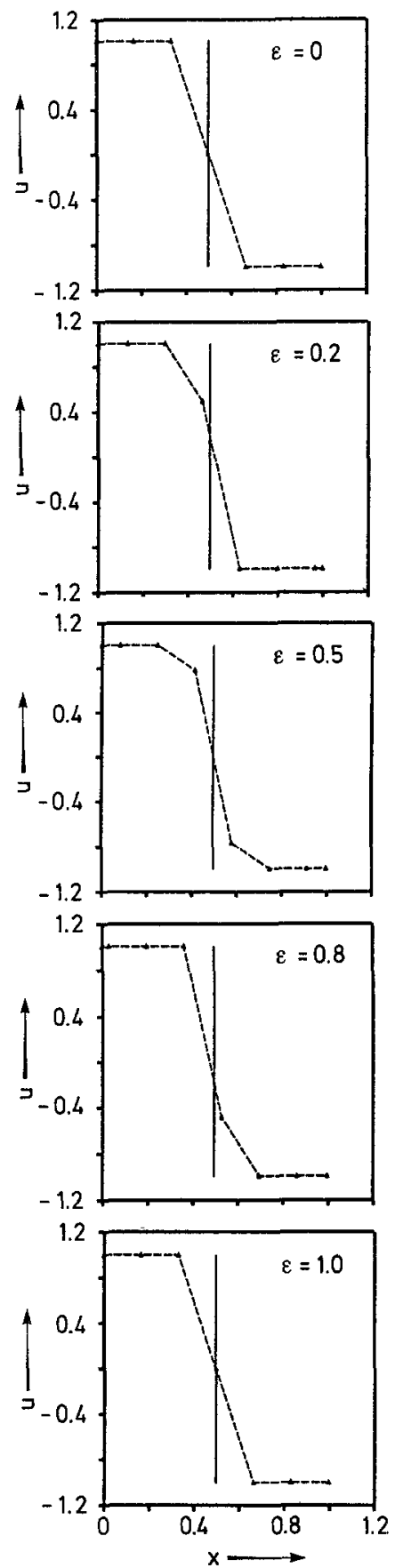

Figs. 6-10. 6 Shock in three cells. 7 Shock in one cell. 8 Locus of intermediate states for shock capturing. 9 Subgrid position of shock. 10 Effect of grid shifting on shock capturing profile

average of the vertex values. The jump relation for a steady discontinuity normal to $\boldsymbol{n}$ reads: $[\boldsymbol{F} \cdot \boldsymbol{n}]=0$, and in the case of a discontinuity along the $y$-direction $\left[\frac{u^{2}}{2}\right]=0$. With the entropy condition stating that $u_{1}>u_{2}$, this becomes $u_{2}=-u_{1}$. Thus, $\boldsymbol{m}=\nabla u /|\nabla u|=-\mathbf{1}_{x}$ and the expression for the frontal speed $\lambda_{m}$ is:

$\lambda_{m}=\lambda_{m} \boldsymbol{m}=(\bar{\lambda} \cdot \boldsymbol{m}) \boldsymbol{m}=\left(\begin{array}{l}\bar{u} \\ 0\end{array}\right)$. 
The capture of a steady shock parallel to the grid is considered, with states $u_{1}=-u_{2}=1$. The grid considered consists in right isoceles triangles, as shown in Fig. 6. The NN strategy, applied to this case, gives:

0 if the triangle is single-target with $\bar{\lambda}=(\bar{u}, 1)^{\tau}$, then send the whole fluctuation to the corresponding downstream node.

- else, look at the gradient-dependent speed $\lambda_{m}=(\bar{u}, 0)^{t}$. Since the triangles of the grid considered are all single-target with $\lambda_{m}$, everything is sent to the corresponding downstream node.

It is clear that such a discontinuity cannot be captured in one cell. Indeed, the fluctuation calculated over a cell whose nodes are at states related by the jump relation is not zero, since the average of $\lambda$ and the gradient of $u$ are non-zero. Therefore a fluctuation will be sent to the downstream node(s), destroying the exact solution as shown in Fig. 7.

One has to consider a shock profile with at least one intermediate state. In the case of Burger's equation, the capture of a shock parallel to the grid is possible within two or three cells. The discussion for a shock profile with two intermediate states $X$ and $Y$ is presented here. It is reasonable to assume a monotonic profile, i.e. $-1 \leqq Y \leqq X \leqq 1$. The grid used in the following discussion is shown in Fig. 6. The conditions for equilibrium to exist are the following: (i) no fluctuation is sent to $(+1)$; (ii) no fluctuation is sent to $(-1)$; (iii) fluctuations to $X$ cancel and (iv) fluctuations to $Y$ cancel. Fluctuations and advection speeds can be easily computed for cells $(11 X)$, $(1 X X),(X X Y),(X Y Y),(Y Y-1)$ and $(Y-1-1)$. The conditions reduce to:

$X \geqq \frac{-1}{2} \quad Y \leqq \frac{1}{2}$

A necessary condition for fluctuations to cancel at nodes $X$ and $Y$ is that the advection speeds in cells $(X X Y)$ and $(X Y Y)$ should be of opposite sign. Thus

$2 Y+X \leqq 0 \quad 2 X+Y \geqq 0$

One can now write the conditions for equilibrium at nodes $X$ and $Y$. These reduce to one, namely,

$2 X^{2}-X Y+2 Y^{2}-3=0 \quad$ or $\quad Y=\frac{X-\sqrt{24-15 X^{2}}}{4}$

The relation can be plotted (Fig. 8) to give the locus of the intermediate states. An interesting result is the symmetric profile $(X=-Y)$, for which the equation yields: $X=-Y=\sqrt{\frac{3}{5}}$

The possible intermediate states lie on the successive curves, $\mathrm{AB}, \mathrm{BC}, \mathrm{CD}$. AB and $\mathrm{CD}$ correspond to a profile with one intermediate state only, $\mathrm{BC}$ corresponds to the two-point profile. Intuitively, it can be assumed that the existence of a 1 or 2 point profile is related to the subgrid position of the shock within the triangle. This result is demonstrated in the following numerical tests.

The domain is defined by $0 \leqq x \leqq 1$ and $0 \leqq y \leqq 1$. The boundary conditions imposed are: $u=1.0$ at $x=0$ and $u=-1.0$ at $x=1$, with initial conditions $u\left(x_{i}, y_{j}\right)=-2 x_{i}+1$. Boundary conditions are such that the exact shock position is at $x=0.5$. A shift of the grid is introduced in the $x$-direction, for different values of a scaling-parameter $\varepsilon$ related to the exact shock location, as shown in Fig. 9. Different profiles are obtained, with one or two intermediate states that lie on the curve ABCD. Cases $\varepsilon=0.0$ and $\varepsilon=0.5$ correspond to a symmetric grid (with respect to the shock's position), and so a symmetric profile may be expected. It is indeed the case with a one-intermediate state profile for $\varepsilon=0.0(Y=0)$ and a two-intermediate-state profile for $\varepsilon=0.5(X=-Y=\sqrt{3 / 5})$. Results are shown in Figs. 10. As can be seen from these figures, the profiles evolve continuously as the relative shock position moves, from a one point profile to a two point profile and then back again to a one point profile, following in effect the theoretical curve ABCD.

In conclusion, shock profiles for the NN scheme contain at most 2 intermediate states. The mechanism by which equilibrium is reached has been demonstrated on this relatively simple case: at steady state, fluctuations sent to each node must "balance" each other. As will be seen in the next section, a similar result is obtained for the Euler system. 


\section{The 2D Euler equations}

Up to now, the fluctuation splitting schemes have been applied to 2D scalar conservation laws. The schemes can also be applied to systems of hyperbolic conservation law such as the Euler equations (Struijs et al. 1991; Struijs et al. 1991).

The starting point is the decomposition of the solution at each time step into a set of linear simple waves, by projection onto the eigenvectors of the flux Jacobian. A multidimensional wave-model is necessary to select the physically meaningful directions of propagation, and the scalar advection schemes discussed previously are used to upwind the splitted residual. A generalized Roe-linearization ensures a conservative discretization.

\subsection{Simple wave solution and multidimensional wave-model}

The 2D Euler equations in quasilinear form,

$\mathbf{U}_{t}+\mathbf{A U}_{x}+\mathbf{B U}_{y}=0 \quad \mathbf{A}=\mathbf{F}_{\mathbf{U}}, \quad \mathbf{B}=\mathbf{G}_{\mathbf{U}}$

admit simple wave solutions of the form

$\mathbf{U}=\mathbf{U}_{0}+\mathbf{R} Q$

where $\mathbf{R}$ is a right-eigenvector of $\mathbf{A} m_{x}+\mathbf{B} m_{y}$, with corresponding eigenvalue $\lambda_{m}$. For each choice of $\boldsymbol{m}$, there exists four eigenvectors corresponding to an entropy wave, a shear wave and a "slow" and "fast" acoustic wave, with respective speeds $\boldsymbol{u} \cdot \boldsymbol{m}, \boldsymbol{u} \cdot \boldsymbol{m}, \boldsymbol{u} \cdot \boldsymbol{m}+\boldsymbol{a}$ and $\boldsymbol{u} \cdot \boldsymbol{m}-\boldsymbol{a}$. Here, $\boldsymbol{a}$ represents the local speed of sound and

$Q=\alpha\left(\boldsymbol{x} \cdot \boldsymbol{m}-\lambda_{\boldsymbol{m}} t\right)+Q_{0}$

with $\alpha$ a constant representing the strength of the wave. $Q$ is governed by the scalar advection equation:

$\frac{\partial Q}{\partial t}+\lambda_{m} \cdot \nabla Q=0$

where $\lambda_{m}$, called the frontal speed, is defined as $\lambda_{m}=\lambda_{m} m$.

More generally, the solution evolves in time from the initial solution at level $n$ as a superposition of simple waves:

$\mathbf{U}=\sum_{k} \alpha_{k}\left(x m_{x}^{k}+y m_{y}^{k}-\lambda_{m}^{k} t\right) \mathbf{R}_{k}+\mathbf{U}_{0}^{n}, \quad \nabla \mathbf{U}=\sum_{k} \alpha_{k} \mathbf{R}_{k} m^{k}$

As shown in Roe (1986), a finite number of those simple waves modelizing elementary flow patterns can be selected to match any linear variation of the data. Algebraic expressions can be derived easily from the decomposition of the vector of primitive variables $\mathbf{W}=(\rho, u, v, p)^{\boldsymbol{\tau}}$ into simple waves, as was done for the vector of conserved variables (33):

$\nabla \mathbf{W}=\sum_{k=1}^{6} \alpha_{k} \mathbf{r}_{k} \boldsymbol{m}_{k}$

where $\mathbf{r}_{k}$ are the right eigenvectors of the flux jacobian in primitive variables. The first so-called wave models (models A and B) consisted of a set of four acoustic waves, propagating normal to each other, an entropy wave propagating in the direction of the entropy gradient, and a modelisation of vorticity (model A) or shear perpendicular to the velocity (model B) to take care of rotational flow. Subsequently, a variant (model C) was constructed, De Palma et al. (1990), with the shear wave propagating in the direction of the pressure gradient. This model has the serious disadvantage that it cannot treat isolated shear layers adequately; also, the acoustic direction is coupled to the shear wave direction. In Roe (1991) and Roe and Beard (1992), starting from the observation that an isolated shock is aligned with the principle axis of the strain rate tensor whereas an isolated shear is at $45^{\circ}$ to it, a more physical model (model D) was suggested which 
effectively sends the shear wave at $45^{\circ}$ to the acoustic direction. In this model, the 6 waves chosen are thus:

$\circ 4$ acoustic waves, with strengths $\alpha_{1}, \alpha_{2}, \alpha_{3}, \alpha_{4}$, propagating in directions: $\theta, \theta+\frac{\pi}{2}, \theta+\pi, \theta+\frac{3 \pi}{2}$

o 1 entropy wave, with strength $\alpha_{5}=\beta$, propagating in the direction $\Phi$

$\circ 1$ shear wave, with strength $\alpha_{6}=\sigma$, propagating in the direction $\psi=\theta \pm \frac{\pi}{4}$.

Substituting in (34), the acoustic direction is found to be precisely the direction of the principle strain rate, while the strength of the shear wave turns out to be the vorticity. The direction of the entropy wave is found to be that of the entropy gradient and the strength its norm. Expressions for the wave strengths and angles are:

$$
\begin{array}{rlrl}
\beta & =\sqrt{\left(\rho_{x}-\frac{p_{x}}{a^{2}}\right)^{2}+\left(\rho_{y}-\frac{p_{y}}{a^{2}}\right)^{2}} & \alpha_{1}=\frac{1}{2}\left[\frac{u_{x}+v_{y}+R}{2}+\frac{p_{x} \cos \theta+p_{y} \sin \theta}{\rho a}\right] \\
\Phi=\arctan _{[0,2 \pi]} \frac{\rho_{y}-\frac{p_{y}}{a^{2}}}{\rho_{x}-\frac{p_{x}}{a^{2}}} & \alpha_{2}=\frac{1}{2}\left[\frac{u_{x}+v_{y}-R}{2}-\frac{p_{x} \sin \theta-p_{y} \cos \theta}{\rho a}\right] \\
\sigma=v_{x}-u_{y}=(\nabla \wedge u) \cdot e_{z} & \alpha_{3}=\frac{1}{2}\left[\frac{u_{x}+v_{y}+R}{2}-\frac{p_{x} \cos \theta+p_{y} \sin \theta}{\rho a}\right] \\
\theta=\frac{1}{2} \arctan _{[0,2 \pi]} \frac{u_{y}+v_{x}}{u_{x}-v_{y}} & \alpha_{4}=\frac{1}{2}\left[\frac{u_{x}+v_{y}-R}{2}+\frac{p_{x} \sin \theta-p_{y} \cos \theta}{\rho a}\right]
\end{array}
$$

$\psi=\theta-\operatorname{sign}(\sigma) \frac{\pi}{4}$

where $R$ is defined as

$$
R=\frac{u_{y}+v_{x}-\sigma \cos 2 \psi}{\sin 2 \theta}=\frac{u_{x}-v_{y}+\sigma \sin 2 \psi}{\cos 2 \theta}
$$

\subsection{Conservative linearization}

This is a multidimensional generalization of Roe's original 1D Flux Difference Splitter (Roe 1981), and is an essential step in that it ensures conservation. Details can be found in Roe et al. (1993) and Deconinck et al. (1991). The main ideas are summarized below:

1. A fundamental assumption is that of piecewise linear data. In $2 \mathrm{D}$, linear elements are triangles. We will therefore consider triangular (unstructured or not) meshes, with unknowns stored at the vertices, as shown in Fig. 11.

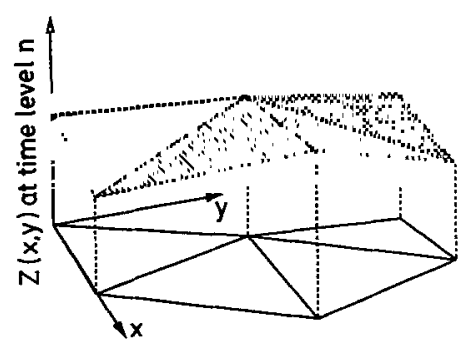

Fig. 11. Continuous piecewise linear data 
2. The vector of unknowns assumed to have linear variation is the parameter vector $\mathbf{Z}=$ $(\sqrt{\rho}, \sqrt{\rho} u, \sqrt{\rho} v, \sqrt{\rho} H)^{\tau} . \mathbf{Z}$ has the property that $\frac{\partial \mathbf{U}}{\partial \mathbf{Z}}, \frac{\partial \mathbf{F}}{\partial \mathbf{Z}}$, and $\frac{\partial \mathbf{G}}{\partial \mathbf{Z}}$ are linear in its components and thus linear over each triangle. All integrals of the above quantities over a triangle therefore reduce to an arithmetic average. The average state $\overline{\mathbf{Z}}$ is thus given by:

$\overline{\mathbf{Z}}=\frac{\mathbf{Z}_{1}+\mathbf{Z}_{2}+\mathbf{Z}_{3}}{3}$

where indices $1,2,3$ denote the vertices of the triangle.

3. One can define average gradients such as:

$\nabla \mathbf{U}=\frac{1}{S_{T}} \iint_{T} \nabla \mathbf{U} d \Omega, \quad \hat{\mathbf{F}}_{x}=\frac{1}{S_{T}} \iint_{T} \mathbf{F}_{x} d \Omega, \quad \hat{\mathbf{G}}_{y}=\frac{1}{S_{T}} \iint_{T} \mathbf{G}_{y} d \Omega$.

As a consequence of the previous assumption,

$\hat{\mathbf{F}}_{x}=\frac{1}{S_{T}} \iint_{T} \frac{\partial \mathbf{F}}{\partial \mathbf{Z}} \mathbf{Z}_{x} d \Omega=\left(\frac{\partial \mathbf{F}}{\partial \mathbf{Z}}\right)_{\overline{\mathbf{Z}}} \mathbf{Z}_{x}$

$\hat{\mathbf{G}}_{y}=\left(\frac{\partial \mathbf{G}}{\partial \mathbf{Z}}\right)_{\overline{\mathbf{Z}}} \mathbf{Z}_{x}, \quad \hat{\mathbf{U}}_{x}=\left(\frac{\partial \mathbf{U}}{\partial \mathbf{Z}}\right)_{\overline{\mathbf{Z}}} \mathbf{Z}_{x}, \quad \hat{\mathbf{U}}_{y}=\left(\frac{\partial \mathbf{U}}{\partial \mathbf{Z}}\right)_{\overline{\mathbf{Z}}} \mathbf{Z}_{y}$.

From the equations above, one obtains:

$\mathbf{Z}_{x}=\left(\frac{\partial \mathbf{U}}{\partial \mathbf{Z}}\right)_{\overline{\mathbf{Z}}}^{-1} \hat{\mathbf{U}}_{x} \quad \mathbf{Z}_{y}=\left(\frac{\partial \mathbf{U}}{\partial \mathbf{Z}}\right)_{\overline{\mathbf{Z}}}^{-1} \hat{\mathbf{U}}_{y}$.

Thus, the fluctuation defined as in (16) can be linearized as

$$
\begin{aligned}
& -\Phi_{T}=\oint_{\partial T} \mathscr{F} \cdot d l=\iint_{T}\left(\mathbf{F}_{x}+\mathbf{G}_{y}\right) d \Omega=S_{T}\left[\hat{\mathbf{F}}_{x}+\hat{\mathbf{G}}_{y}\right] \\
& \quad=S_{T}\left[\left(\frac{\partial \mathbf{F}}{\partial \mathbf{Z}}\right)_{\overline{\mathbf{z}}}\left(\frac{\partial \mathbf{Z}}{\partial \mathbf{U}}\right)_{\overline{\mathbf{Z}}} \hat{\mathbf{U}}_{x}+\left(\frac{\partial \mathbf{G}}{\partial \mathbf{Z}}\right)_{\overline{\mathbf{Z}}}\left(\frac{\partial \mathbf{Z}}{\partial \mathbf{U}}\right)_{\overline{\mathbf{Z}}} \hat{\mathbf{U}}_{y}\right] \\
& =S_{T}\left[\left(\frac{\partial \mathbf{F}}{\partial \mathbf{U}}\right)_{\overline{\mathbf{Z}}} \hat{\mathbf{U}}_{x}+\left(\frac{\partial G}{\partial \mathbf{U}}\right)_{\overline{\mathbf{z}}} \hat{\mathbf{U}}_{y}\right] .
\end{aligned}
$$

The linearization is conservative in two senses:

(1) $\sum_{T} S_{T}\left[\hat{\mathbf{F}}_{x}+\hat{\mathbf{G}}_{y}\right]=\sum_{T} \oint_{\partial T} \mathscr{F} \cdot d l=\oint_{\partial \Omega_{\text {outer }}} \mathscr{F} \cdot d l$

(2) $\hat{\mathbf{F}}_{x}+\hat{\mathbf{G}}_{y}=\left[\left(\frac{\partial \mathbf{F}}{\partial \mathbf{U}}\right)_{\overline{\mathbf{Z}}} \hat{\mathbf{U}}_{x}+\left(\frac{\partial \mathbf{G}}{\partial \mathbf{U}}\right)_{\overline{\mathbf{Z}}} \hat{\mathbf{U}}_{y}\right]$.

The first statement characterizes the "telescoping property" of the residuals: the only terms left after summation involve the outer boundaries of the domain. The second statement shows that for each triangle the quasilinear form of the flux divergence is an exact expression for the flux divergence. This will prove essential in the next step.

4. From the wave decomposition of the gradient of $\mathbf{U}$ at the average state $\overline{\mathbf{Z}}$,

$\nabla \mathbf{U}=\sum_{k=1}^{6} \bar{\alpha}_{k} \overline{\mathbf{R}}_{k} \boldsymbol{m}_{k}$

and using the property stated above, one has an exact expression for the flux divergence:

$\hat{\mathbf{F}}_{x}+\hat{\mathbf{G}}_{y}=\sum_{k=1}^{6} \bar{\alpha}_{k} \bar{\lambda}_{m}^{k} \overline{\mathbf{R}}_{k}$. 
5. Thus the expression for the conservative residual over triangle $T$ is:

$\Phi_{T}=-\oint_{\partial T} \mathscr{F} \cdot d l=-S_{T} \sum_{k=1}^{6} \bar{\alpha}_{k} \bar{\lambda}_{m}^{k} \overline{\mathbf{R}}_{k}=\sum_{k=1}^{6} \Phi_{T}^{k}$

6. Because of the assumption that $\mathbf{Z}$ is linear over the triangle, the consistent gradients of the primitive variables, needed to compute the wave strengths and angles, Eq. (34), are given by:

$\nabla \mathbf{W}=\left(\frac{\partial \mathbf{W}}{\partial \mathbf{Z}}\right)_{\overline{\mathbf{Z}}} \nabla \mathbf{Z}$.

\subsection{Fluctuations and speeds}

The residual in cell $T$ has been split into 6 wave contributions. Each wave is associated to an advection equation:

$\frac{\partial \bar{Q}^{k}}{\partial t}+\bar{\lambda}_{m}^{k} \cdot \nabla \bar{Q}^{k}=0$

where $\bar{Q}^{k}(x, y, t)=\bar{\alpha}_{k}\left(\boldsymbol{x} \cdot \boldsymbol{m}_{k}-\bar{\lambda}_{m}^{k} t\right)+Q_{0}^{k}$ and $\nabla \bar{Q}^{k}=\bar{\alpha}_{k} \boldsymbol{m}_{k}$. For each wave, one can define the scalar fluctuation and the residual in conservative variable as:

$-\Psi_{T}^{k}=\iint_{T} \lambda_{m}^{k} \cdot \nabla \bar{Q}^{k} d \Omega, \quad \Phi_{T}^{k}=\Psi_{T}^{k} \overline{\mathbf{R}}_{k}$

and the general update formula takes the form

$\mathbf{U}_{i}^{n+1}=U_{i}^{n}+\frac{\Delta t}{S_{i}} \sum_{T} \sum_{k=1}^{6} \beta_{T}^{i, k} \Phi_{T}^{k}$

As before, one has the important property, that $\forall \bar{\lambda}^{k}$ :

$\bar{\lambda}_{m}^{k}=\left(\bar{\lambda}_{k} \cdot m_{k}\right) \boldsymbol{m}_{k}, \quad-\Psi_{T}^{k}=S_{T} \bar{\lambda}_{m}^{k} \cdot \nabla \bar{Q}^{k}=S_{T} \bar{\lambda}^{k} \cdot \nabla \bar{Q}^{k}=S_{T} \bar{\lambda}_{m}^{k} \bar{\alpha}_{k}$.

The fluctuation is not changed whether one takes the speed $\bar{\lambda}^{k}$ or the gradient-dependent speed $\bar{\lambda}_{m}^{k}$. The physical convection speeds for the waves are the $\bar{\lambda}^{k}$, called "ray speeds", and are given by the velocity and the bicharacteristic vector associated to $m$ :

$\lambda_{e}=u, \quad \lambda_{s}=u, \quad \lambda_{a}=u+a m$

whereas the gradient-dependent speeds correspond to the actual eigenvalues of the matrix $\mathbf{A} m_{x}+\mathbf{B} m_{y}$, and are known as "frontal speeds":

$\lambda_{m_{e}}=(\boldsymbol{u} \cdot \boldsymbol{m}) \boldsymbol{m}, \quad \lambda_{m_{s}}=(\boldsymbol{u} \cdot \boldsymbol{m}) \boldsymbol{m}, \quad \lambda_{m_{a}}=(\boldsymbol{u} \cdot \boldsymbol{m}+a) \boldsymbol{m}$.

The choice of the ray speeds as the full convection speeds is the physical choice, since it corresponds to taking the speeds within the cone of dependence, as shown in Fig. 12 for the case of a supersonic flow. Thus information cannot be convected outside the domain of influence of the pertubation, delimited by the Mach angle $\mu=\sin ^{-1}(1 / M)$.

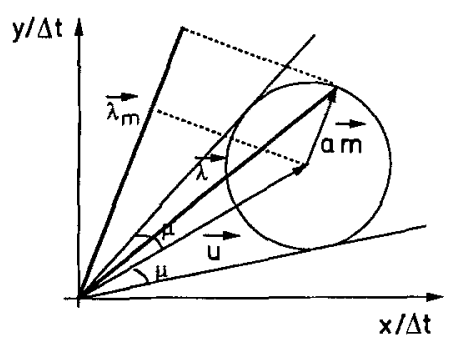

Fig. 12. Acoustic ray speed and frontal speed 
In conclusion, the linear schemes of Sect. 3.1 only make use of the speeds (40), while the non-linear schemes also need the speeds (41) for the two-target triangles.

\section{Numerical results}

Some numerical results are presented here that show perfect capturing of discontinuities aligned with the grid. A jet-interaction case is also computed and compared to a TVD finite volume scheme.

\subsection{Normal shock}

A normal shock is computed: states $A$ and $B$ related by the Rankine-Hugoniot relations are imposed at the inlet and outlet, and periodic boundary conditions imposed at other boundaries. All advection schemes reduce to the 1-target formula. As for the scalar example, capturing occurs with two intermediate states $X$ and $Y$, thus enforcing an entropy condition forbidding the existence of expansion shocks. Figure 13 shows the Mach line contours, Fig. 14 shows a density crosssection.

The mechanism by which the shock is trapped is similar to that studied previously for the scalar conservation law. This is illustrated fy Figs. 15-17, representing the wave speeds scaled by the absolute value of their strengths: waves moving away from the discontinuity have zero strength and those moving towards the discontinuity cancel one another.
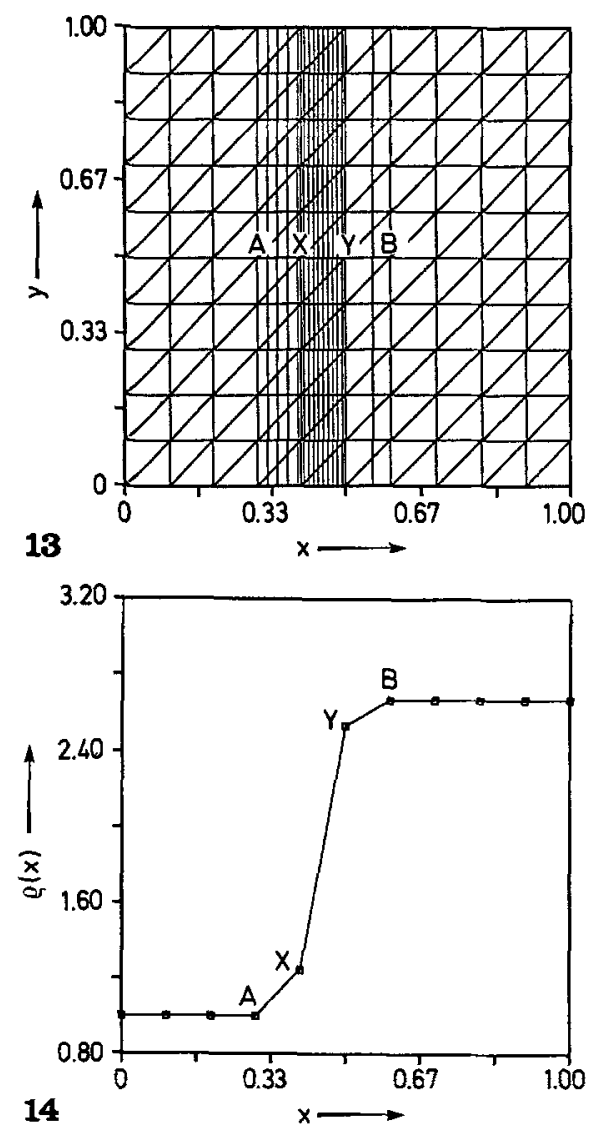

15

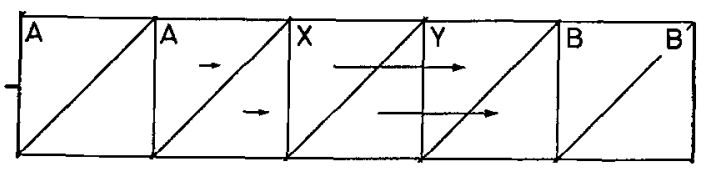

16

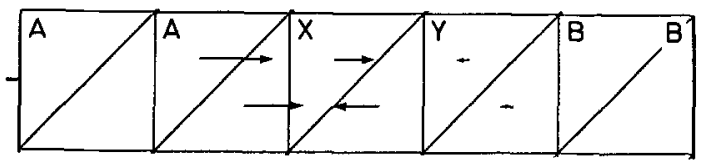

17

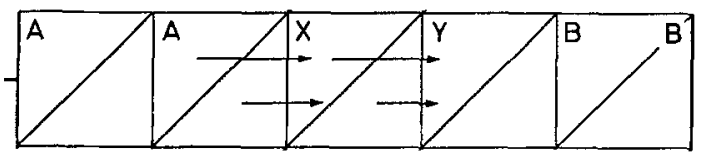

Figs. 13-17. 13 Normal shock: Mach line contours. 14 Normal shock: density cross-section. 15 Acoustic wave " $\lambda_{+}=u+a "$ ". 16 Acoustic wave " $\lambda_{-}=u-a "$. 17 Entropy wave " $\lambda_{e}=u$ " 

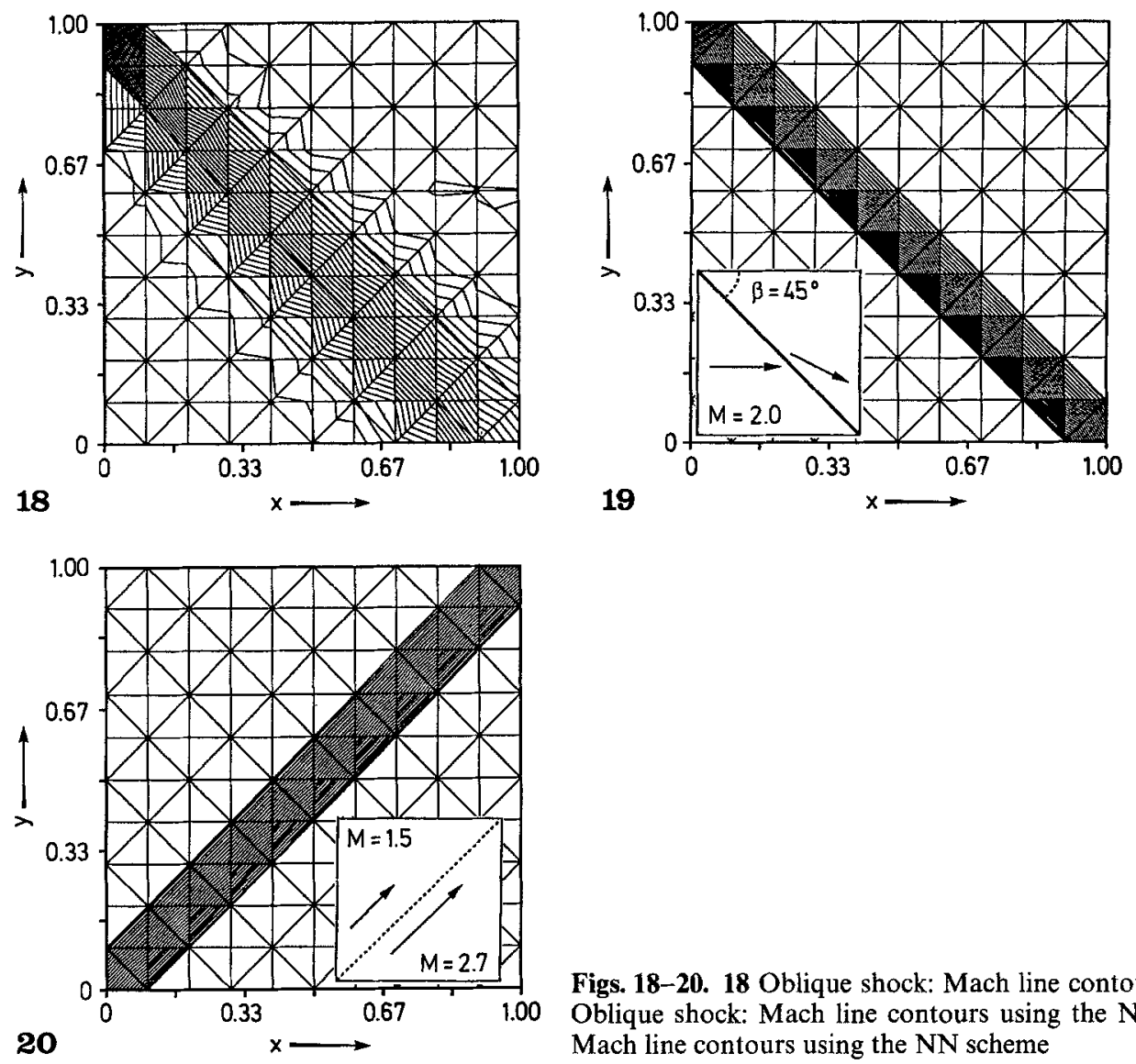

Figs. 18-20. 18 Oblique shock: Mach line contours using the N scheme. 19 Oblique shock: Mach line contours using the NN scheme. 20 Shear layer: Mach line contours using the NN scheme
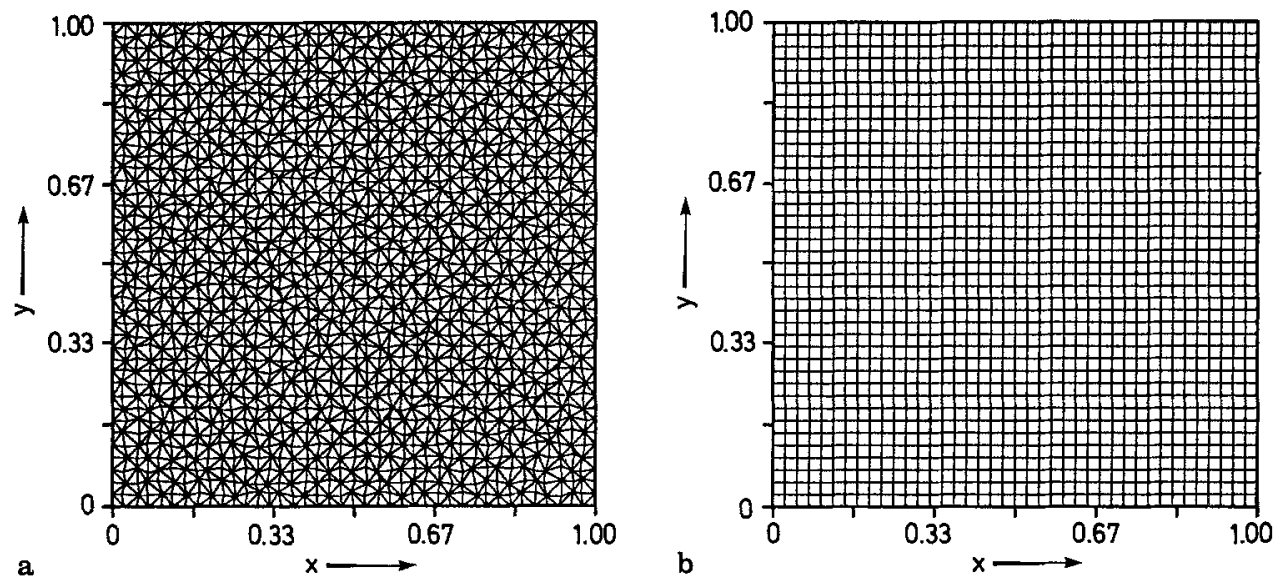

Fig. 21. a Randomized triangular mesh, b finite volume grid

\subsection{Oblique shock}

Starting from a state $A$ with speed aligned with the horizontal axis, an oblique shock at $45^{\circ}$ is computed on an isotropic grid. The solution computed with the $\mathrm{N}$ scheme is smeared, whereas the non-linear scheme (NN) captures the discontinuity perfectly, in two cells (Figs. 18 and 19). This is easily explained from the theory by comparing the directions of the speeds used in the two schemes. 

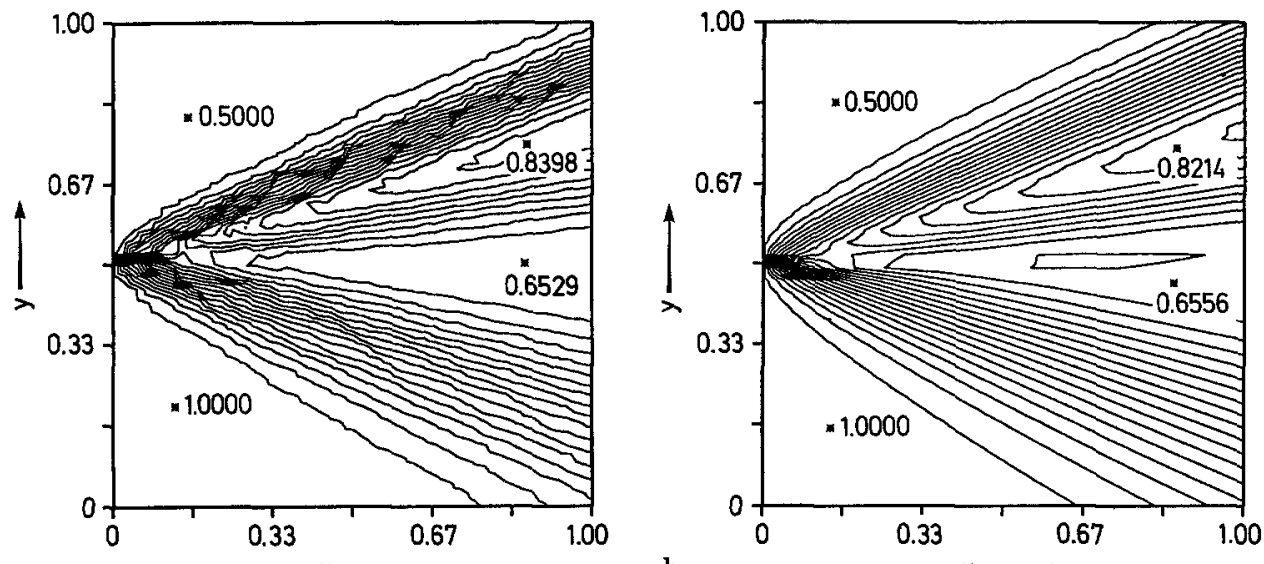

a

Fig. 22. Density line contours: a $\mathrm{N}$ scheme, b Roe $1^{\text {st }}$ order
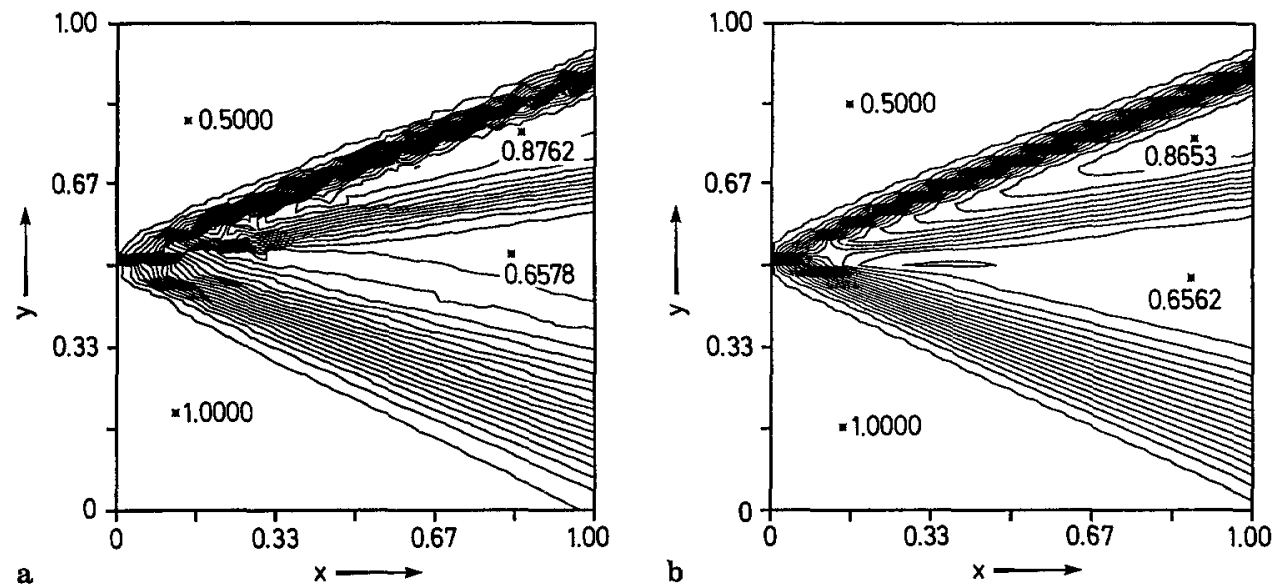

Fig. 23. Density line contours: a PSI scheme, b Roe $2^{\text {nd }}$ order

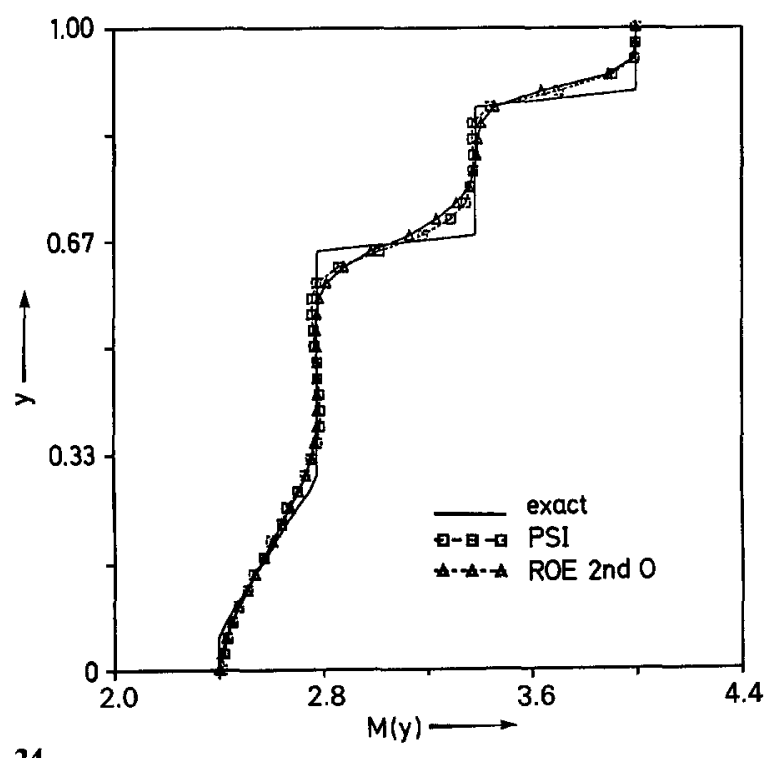

24

Fig. 24 and 25. 24 Mach number cross-section: exact/PSI/Roe $2^{\text {nd }} \mathcal{O} .25$ Convergence histories

25

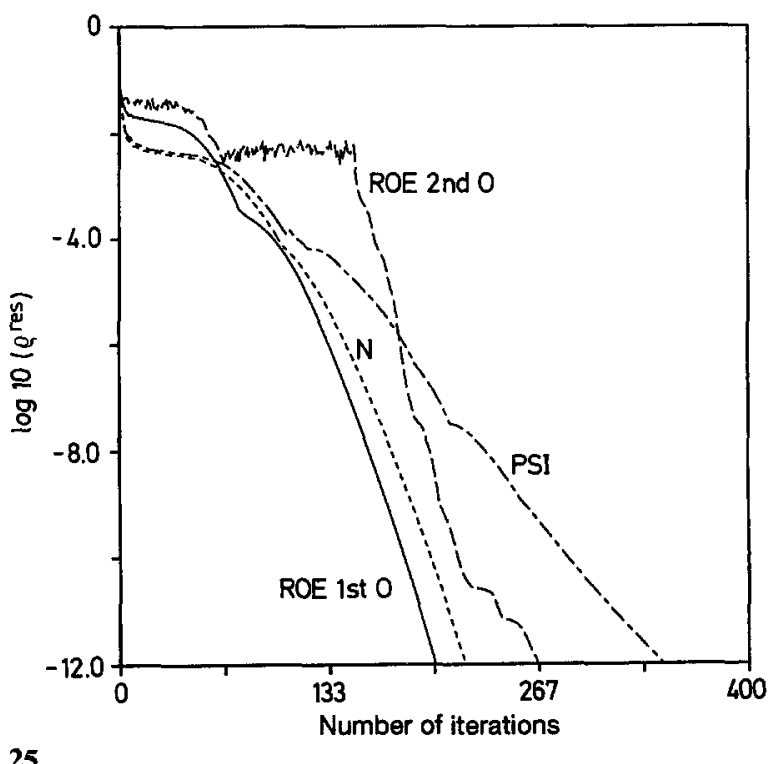




\subsection{Shear layer}

A supersonic shear at $45^{\circ}$ with the $x$-axis is computed on the same isotropic grid: stream 1 is characterized by $M_{1} \approx 2.2, \rho_{1}=1.0$ and stream 2 by $M_{2} \approx 3.8, \rho_{2}=2.0$. The shear is captured by the non-linear scheme in two rows on the isotropic grid, as shown in Fig. 20. As for the oblique shock, the linear schemes cannot preserve the shear on this mesh.

\subsection{Supersonic jet interaction}

The interaction of two parallel jets is computed: the upper stream has conditions $M_{1}=4.0$, $\rho_{1}=0.50$ and $p_{1}=0.25$; the lower stream has conditions $M_{2}=2.4, \rho_{2}=1.0$ and $p_{2}=1.0$. Their interaction produces a shock wave propagating in the low pressure region and an expansion fan propagating in the high pressure region. A contact is also produced. Four numerical solutions were computed: on a randomized $40 \times 40$ triangular mesh (Fig. 21a), two solutions using the linear $\mathrm{N}$ and the non-linear PSI (similar to the NN scheme discussed here) schemes, and on a $40 \times 40$ Cartesian finite volume grid (Fig. 21b), a first and second order solution using Roe's Approximate Riemann solver. The second order solution was computed using gradient reconstruction with a minmod-type limiter (Barth 1990). The limiter was frozen after 150 iterations.

Figures 22 and 23 show density line contours for the four computations. As can be seen, the accuracy of the $\mathrm{N}$ scheme lies somewhat between first and second order. As for the non-linear scheme, the oblique shock and shear are captured slightly more sharply than by the second-order Roe scheme, see Fig. 24.

Figure 25 shows the four convergence histories, i.e. the maximum density residual over all nodes versus the number of time-steps, corresponding to a Runge-Kutta 4 stage scheme with $\mathrm{CFL}=2.0(\mathrm{~N}, \mathrm{PSI})$ and $\mathrm{CFL}=1.0\left(\right.$ Roe $1^{\text {st }}$ and $\left.2^{\text {nd }} \mathcal{O}\right)$. All schemes converge to machine accuracy.

Note that the computational stencil in the multidimensional computation involves only the vertices of triangles meeting at a given point.

\section{Concluding remarks}

In this contribution we have surveyed some efforts made during the last years to build a multidimensional theory for upwind schemes. These ideas are conceptually not different from the successfull upwind solvers developed in the eighties, as far as one dimensional flow is concerned.

The theory has been rebuilt only in the way the extension to two dimensions is handled, by curing the weakness of the one dimensional physical modelling used in the state of the art upwind methods.

The new approach has a strong potential, especially for codes based on triangles and tetrahedra, bringing these unstructured grid methods which suffer most from the one-dimensional modelling to the same level of capability as structured grid solvers: perfect capturing of shears and shocks aligned with one set of cell boundaries, and high resolution on very compact stencils. Work remains to be done for improving wave models and boundary conditions, especially for subsonic flows.

\section{Acknowledgements}

The work at VKI was supported by the Commission of the European Community under Contract AERO-0003-C in Area 5 (Aeronautics) of the BRITE/EURAM Program (1989-1993).

\section{References}

Barth, T. J. (1990): On unstructured grids and solvers. VKI LS 1990-04

Bourgois, G.; Deconinck, H.; Roe, P. L.; Struijs, R. (1992): Multidimensional upwind schemes for scalar advection on tetrahedral meshes. First European Computational Fluid Dynamics Conference, Brussels 
Deconinck, H.; Roe, P. L.; Struijs, R. (1991): A multidimensional generalization of Roe's flux difference splitter for the Euler equations. 4th ISCFD Conference, U.C. Davis

De Palma, P.; Deconinck, H.; Struijs, R. (1990): Investigation of Roe's 2D wave models for the Euler equations. VKI TN 172

Roe, P. L. (1981): The use of the Riemann problem in finite differences. In: Reynolds, W. C.; MacCormac, R. W. (eds): 7 th Int. Conf. Numerical Meth. in Fluid Dynamics. Berlin, Heidelberg, New York: Springer

Roe, P. L. (1981): Approximate Riemann solvers, parameter vectors, and difference schemes. J. Comp. Phys. 43, No. 2, 357

Roe, P. L. (1982): Fluctuations and signals-A framework for numerical evolution problems. In: Mortan, K. W.; Baines, M. J. (eds): Numerical Methods for Fluid Dynamics. New York: Academic Press

Roe, P. L. (1986): Discrete models for the numerical analysis of time-dependent multidimensional gas dynamics. J. Comp. Phys. $63,458-476$

Roe, P. L. (1987): Linear advection schemes on triangular meshes. CoA Report No. 8720, Cranfield, November

Roe, P. L. (1991): Beyond the Riemann problem I. ICASE-NASA Langley workshop. September

Roe, P. L.; Beard, L. (1992): An improved wave model for multidimensional upwinding of the Euler equations. 13th International Conference on Numerical Methods in Fluid Dynamics. Rome

Roe, P. L.; Struijs, R.; Deconinck, H. (1993): A conservative linearization for the multidimensional Euler equations. To appear in J. Comp. Phys.

Sidilkover, D. (1990): Numerical solution to steady-state problems with discontinuities. Ph.D. thesis; Wiezmann Institute, Israel

Struijs, R.; Deconinck, H.; Roe, P. L. (1991): Fluctuation splitting schemes for the 2D Euler equations. VKI lecture series 1991-01

Struijs, R.; Deconinck, H.; dePalma, P.; Roe, P. L.; Powell, K. G. (1991): Progress on multidimensional upwind Euler solvers for unstructured grids. AIAA 91-1550

Communicated by T. E. Tezduyar, July 3, 1992 